\title{
Application of a Systematic Protocol in the Treatment of TMDs With Occlusal Appliances: Effectiveness and Efficiency in a Longitudinal Retrospective Study With Medium-Term Follow-Up
}

\section{Doria Tolevski Meshkova, Paola Di Giacomo, Fabrizio Panti, Anna D’Urso, Emanuela Serritella, Carlo Di Paolo}

Gnathologic Division, Department of Oral and Maxillo-Facial Sciences, Policlinico Umberto I, "Sapienza" University of Rome, Rome, Italy

Received : 27-02-19.

Accepted : 17-05-19.

Published : 06-08-19.
Objectives: This study aimed to assess effectiveness, efficiency, and feasibility of a systematic protocol for the choice and management of occlusal splints (OA) in the treatment of temporomandibular disorders (TMDs). Materials and Methods: A longitudinal retrospective study was conducted. Two different samples, G1 + G2 (337 patients), between January 2011 and January 2014, were selected according to inclusion and exclusion criteria. G1 was composed of patients visited at Policlinico Umberto I, Head-Neck Department, Sapienza University of Rome, Italy, and patients in G2 visited at a private structure in Rome. Pain records and functionality were compared before (T0) and at the end of therapy (T1). A follow-up group, composed of 100 patients randomly selected among those who completed treatment for at least 1 year (T2), was analyzed and symptomatology records were compared. Descriptive statistical analysis was performed. Results: In the entire sample, joint and muscular pain, joint noises and mandibular functionality, headache, and neck pain improved from T0 to T2. The average time for articular and muscular recovery was 6.4 months. Comparing treatment outcomes, there were not statistically significant differences between the two groups. Conclusions: Treatment outcomes using this setting of protocol showed a positive trend also in the medium term. The use of a systematic protocol seems to reduce operator-dependent factors.

KeYwords: Occlusal appliances, systematic protocol, temporomandibular disorders

\section{INTRODUCTION}

$\neg$ emporomandibular disorders (TMDs) are a 2 heterogeneous group of skeletal, articular, and neuromuscular conditions involving masticatory muscles, temporomandibular joint, and associated structures. ${ }^{[1]}$

TMDs are the most common cause of orofacial pain of nonodontogenic origin and the second most common musculoskeletal condition of pain and disability after chronic low-back pain..$^{[2]}$

These disorders are well established for what concerning the scientific classification and from a diagnostic point of view. ${ }^{[3]}$ Recommended treatments are conservative

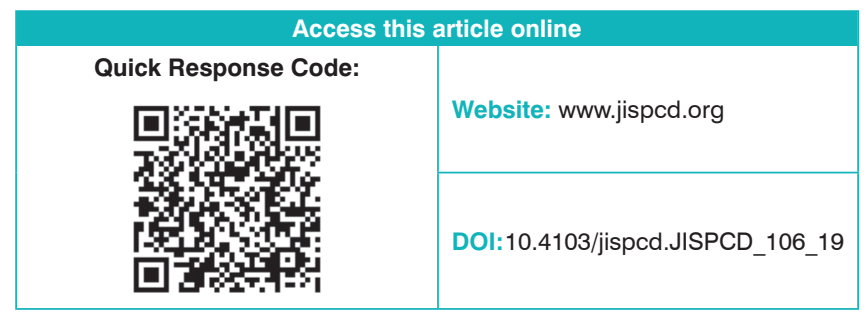

and occlusal splints are the most common therapy among these. ${ }^{[4]}$

Occlusal splints are the most common therapy among conservative treatments. ${ }^{[5]}$

There are a huge variety of occlusal devices used for the most disparate indications and there is a consequent confusion on the actual effectiveness of each appliance. $^{[6]}$

Address for correspondence: Dr. Paola Di Giacomo, Via Caserta 6, 00161 Rome, Italy.

E-mail: p.digiacomo@uniroma1.it

This is an open access journal, and articles are distributed under the terms of the Creative Commons Attribution-NonCommercial-ShareAlike 4.0 License, which allows others to remix, tweak, and build upon the work non-commercially, as long as appropriate credit is given and the new creations are licensed under the identical terms.

For reprints contact: reprints@medknow.com

How to cite this article: Tolevski Meshkova D, Di Giacomo P, Panti F, D’Urso A, Serritella E, Di Paolo C. Application of a systematic protocol in the treatment of TMDs with occlusal appliances: Effectiveness and efficiency in a longitudinal retrospective study with medium-term follow up. J Int Soc Prevent Communit Dent 2019;9:372-82. 
Almost all current scientific guidelines suggest conservative treatments, planned on the basis of a specific clinical diagnosis. ${ }^{[7]}$

The authors of this study have been applying for years a treatment protocol for the use of occlusal appliances, based on what observed during the diagnostic process and on a customized management, in relation to the characteristics of the patient and the type of pathology. This protocol has been revised and updated over time on the basis of new literature indications and on the presence of the new Diagnostic Criteria for Temporomandibular Disorders criteria (DC/TMD) $)^{[3]}$ instead of Research Diagnostic Criteria for TMD.

A longitudinal retrospective study was performed on a large and selected sample with the purpose to evaluate the medium long-term effectiveness and efficiency of this protocol, in response to the need to help the specialist to deal with the care of dysfunctional patients (adults and growing) with clarity and predictability. Clinical outcomes emerging from the research were examined using descriptive statistical analysis. Chi-squared test was performed with the purpose to compare results stemming from two different samples (one public and the other private) in order to evaluate if the protocol was feasible for any clinician. The research was not intended to compare different types of patients/ therapies because it was not a case-control study.

Other types of conservative treatments for TMDs such as physical rehabilitation or pharmacologic treatments were not considered in order not to create misleading factors. However, it is clear that normally occlusal splints are applied along with other conservative therapies (such as those mentioned earlier).

\section{Materials and Methods \\ Study design}

This longitudinal retrospective study was conducted over a period of 1 year (May 2017 to May 2018) on individuals with TMDs treated at the Head-Neck Department, Sapienza University of Rome, Italy, and at a private dental structure in Rome between January 2011 and January 2014.

The research was approved by the Institutional Human Ethics Committee, Sapienza University of Rome (Protocol No. 349).

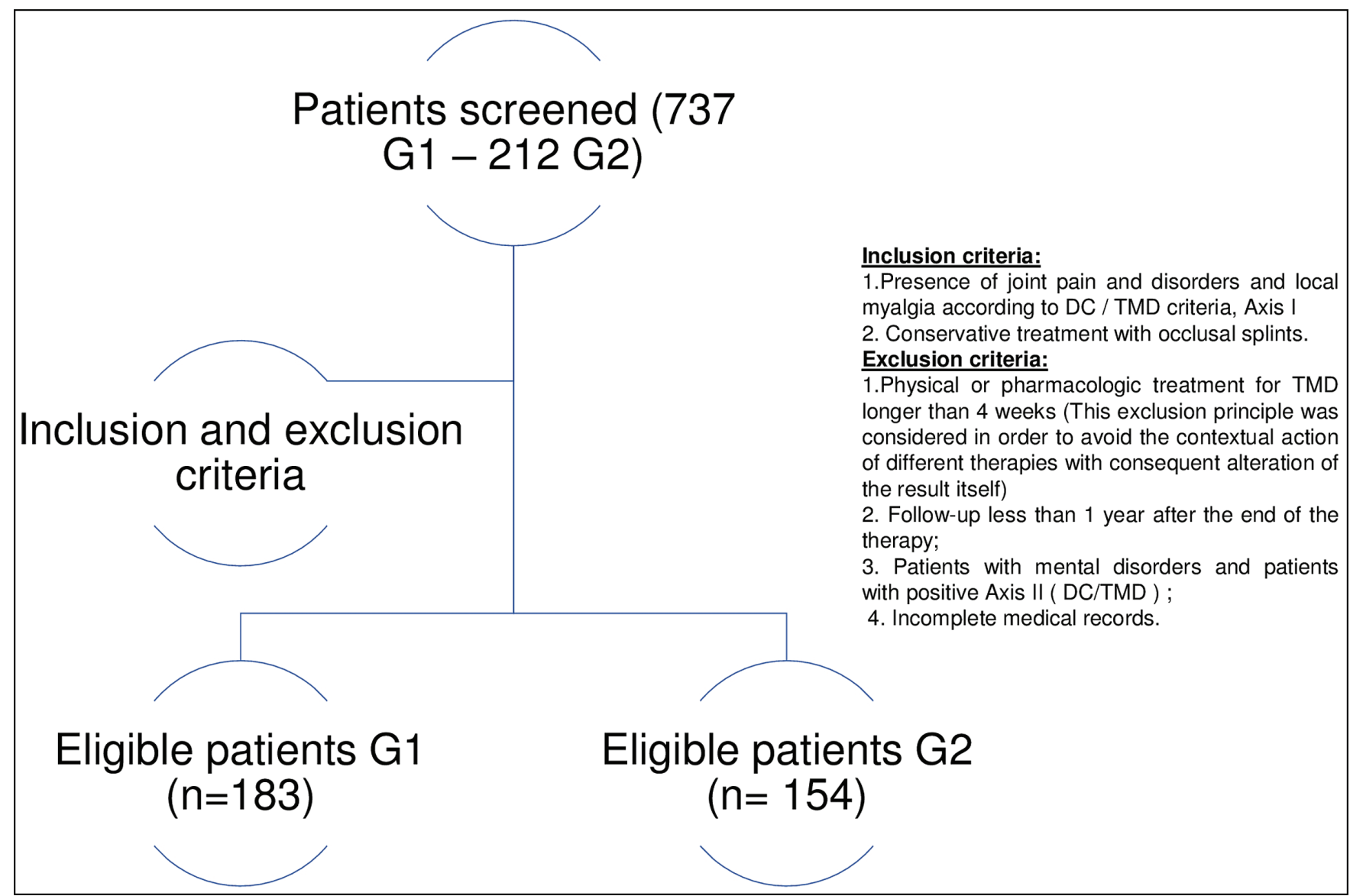

Figure 1: Flow of patients through the study 
Inclusion and exclusion criteria and the flow of patients through the study are indicated in Figure 1.

Sample size was calculated using Cochran's statistical formula at $95 \%$ confidence interval:

$$
\mathrm{n}=\frac{\mathrm{n} 0}{1+[(\mathrm{n} 0-1) / N]}
$$

The sample composed of 337 patients was representative of the screened TMD population $(n=275$ is the minimum sample size required $\mathrm{N}=949$ is the screened TMD population).

The first group of patients (G1), selected at Policlinico Umberto I, Gnathologic Division, Sapienza University of Rome, was approached by various calibrated operators. The second one (G2), selected at a private specialized structure in Rome, was visited by a single expert clinician. The rationale was to verify the applicability of the treatment protocol by any operator. In order to avoid selection bias, both groups were homogenous by age, gender, and pathology.

Subjects eligible for the study had provided signed informed consent, according to the World Medical Association's Declaration of Helsinki.

In order to evaluate the results in the medium term, a sample of 100 patients (GF: Follow-Up Group) was selected among those who had completed treatment for at least 1 year. Fifty patients of G1 and 50 patients of G2 were selected by simple random sampling using a

\begin{tabular}{|c|c|}
\hline Personal Data & gender, age (divided into 6 categories: $0-20,20-30,30-40,40-50,50-60,60-80$ ), telephone number and/or e-mail \\
\hline Pathology & (according to the DC / TMD classification); \\
\hline $\begin{array}{l}\text { Type of occlusal } \\
\text { splint }\end{array}$ & Di.T.R.A., RA.DI.CA., Michigan splint \\
\hline $\begin{array}{l}\text { Pain (VNS) } \\
\text { evaluated at T0, } \\
\underline{1}\end{array}$ & $\begin{array}{l}\text { joint pain, muscle pain, divided into } 4 \text { categories based on the value conferred in terms of scale NVS (Numeric Verbal } \\
\text { Scale): mild }(0-20) \text {; moderate (20-50); strong }(50-80) \text {; severe }(80-100) \text {. }\end{array}$ \\
\hline $\begin{array}{l}\text { Mandibular } \\
\text { functionality } \\
\text { evaluated at T0, } \\
\text { T1 }\end{array}$ & $\begin{array}{l}\text { 1. Presence/absence of articular noises compatible with a disk dislocation with reduction; } 2 \text {. Measurement of the } \\
\text { maximum spontaneous mouth opening considering } 40 \mathrm{~mm} \text { as a standard limit taking into account the present skeletal } \\
\text { state. These two parameters were considered together, obtaining } 4 \text { categories of evaluation of the mandibular function: } \\
\text { physiological (absence of noise and normal opening); good (occasional noise and normal opening); sufficient (constant } \\
\text { noise and normal opening); insufficient (constant and/or absent noise and aperture less than } 40 \mathrm{~mm} \text { ); }\end{array}$ \\
\hline $\begin{array}{l}\text { TMD } \\
\text { Comorbidities } \\
\text { evaluated at T0, } \\
\underline{\text { T1 }}\end{array}$ & $\begin{array}{l}\text { Analysis of Headache and Neck pain using NVS (Numeric Verbal Scale): mild (0-20); moderate (20-50); strong (50-80); } \\
\text { severe (80-100). }\end{array}$ \\
\hline $\begin{array}{l}\text { Duration of } \\
\text { therapy }\end{array}$ & (expressed in months) divided into the following categories: 0-10, 10-20, 20-30 and 30-40. \\
\hline $\begin{array}{l}\text { Improvement } \\
\text { time }\end{array}$ & (minimum time in which improvements were obtained) \\
\hline $\begin{array}{l}\text { Verification of } \\
\text { side effects on } \\
\text { the occlusion }\end{array}$ & assessed both subjectively (perceived by the patient) and objectively (highlighted by the clinician). \\
\hline
\end{tabular}

Figure 2: Data evaluated in medical records 
randomization software STATA (StataCorp, College Station, TX).

Medical records of the patients belonging to the selected samples were examined by a single operator, which had no direct relationship either with the public or private structure [Figure 2]. This researcher did not know which participants belonged to groups $\mathrm{G} 1$ or $\mathrm{G} 2$.

\section{TREATMENT PROTOCOL}

Patients of this study were treated with occlusal splints, according to the diagnosis and the characteristics of the patient, as indicated by the literature and guidelines of Sapienza School of Gnathology [Figures 3 and 4]. ${ }^{[7-10]}$
These occlusal devices [Figure 3] are indicated also toward TMD comorbidities such as headache and neck pain. ${ }^{[8-10]}$

Among the alternatives indicated by the scientific literature, on the basis of studies conducted over time, Michigan splint for muscular dysfunctions, DI.TRA, and RA.DI.CA for articular ones were chosen.

The management protocol included preparatory and progressive phases that modified the duration of daily application of the occlusal splints up to a minimum use, no later than 12 months.

In order to allow an individualized management, other criteria were considered [Figure 4].

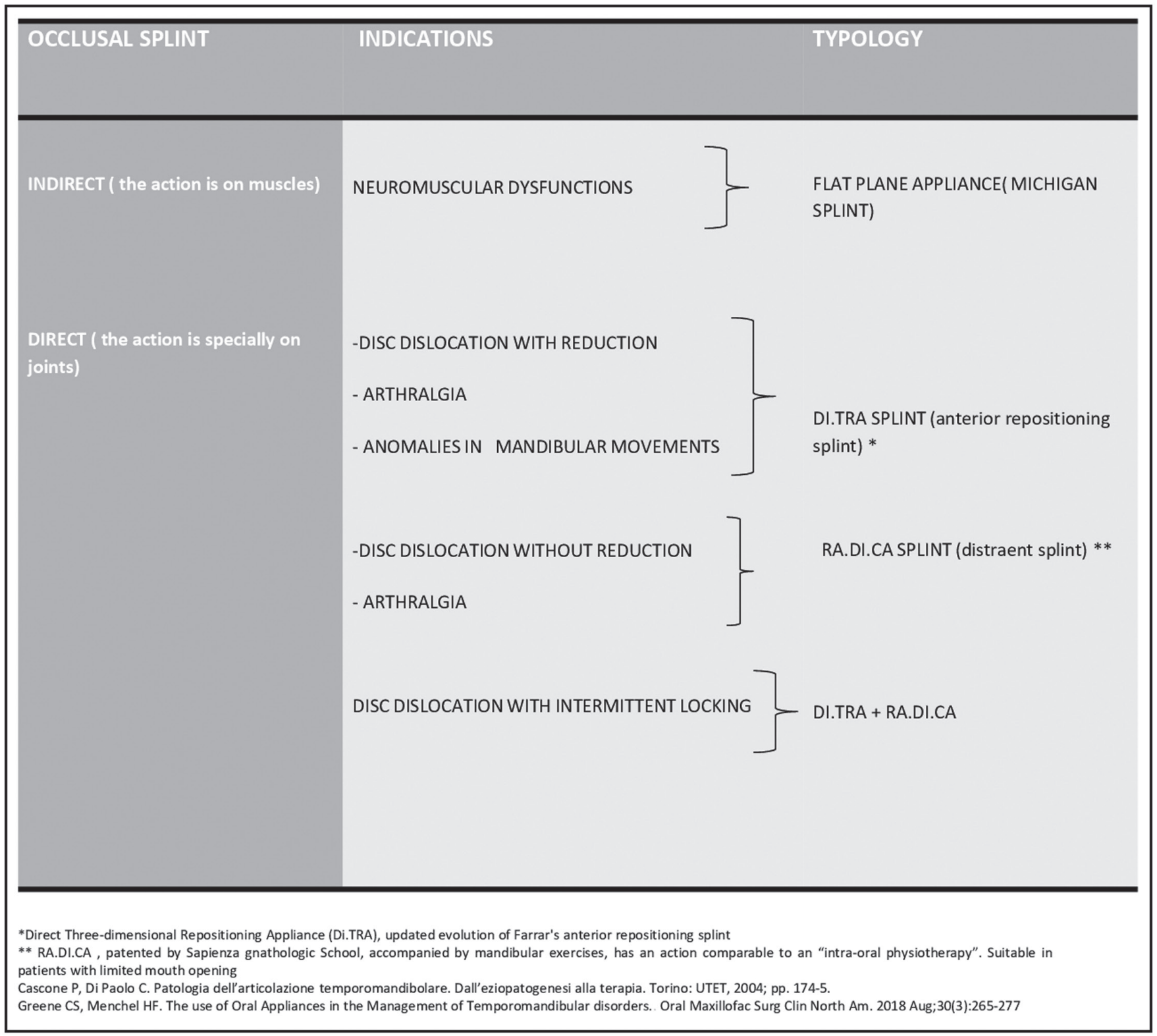

Figure 3: Flowchart for the choice of the occlusal splints, according to diagnosis 


\section{Data collection}

Data regarding symptomatology, both algic and functional, were compared before (T0), at the end of therapy (T1), and during the follow-up.

The quantitative data concerning the results at the end of therapy (T1) and during the follow-up (T2), moreover, were translated into a qualitative measure [Figure 5].

\section{Statistical ANALYsis}

Statistical software STATA was used for data analysis. Descriptive statistical analysis was performed. Percentage, average, median, mode, standard deviation, minimum, and maximum value of each collected data were performed.

Chi-squared test was performed in order to compare general treatment outcomes of the two groups, with a level of statistical significance $\alpha=0.05,3$ degrees of freedom. Confidence intervals were set at $95 \%$.

\section{Results}

Only the most significant results are reported.

Results concerning personal data, type of DC/TMD disorder, and type of splint are shown in Table 1.

\section{Effectiveness: treatment outcomes in G1 and G2 at} $\mathrm{T} 0, \mathrm{~T} 1$, and $\mathrm{T} 2$

Joint pain: At T1, the category mild pain ( $0-20$ verbal numeric scale (VNS)) was prevalent; $80 \%$ (270) of patients, of whom $74 \%$ (248 patients) reported total absence of pain and 6\% (22 patients) reported it as discomfort. At T2, the category mild pain (0-20 VNS) was predominant for $90 \%$ (69) of patients.

Muscular pain: At T1, the category mild pain (0-20 VNS) was prevalent; $75 \%$ (253) of patients, of whom $65 \%$ (219 patients) reported total absence and 10\% (34 patients) reported it as discomfort. At T2, the category mild pain (0-20 VNS) was predominant for 90\% (69) of patients.

Comorbidities improved but with results less significant.

The comparison of data obtained at $\mathrm{T} 0, \mathrm{~T} 1$, and $\mathrm{T} 2$ showed a marked positive change, in terms of both quantitative and qualitative evaluations, not only concerning algic symptomatology but also functionality (improvement in mouth opening and reduction of joint noises).

Joint noises: At $\mathrm{T} 0$, joint noises compatible with disc dislocation with reduction were present in 39\% (131) of the patients, of which $14 \%$ (47) were episodic and $25 \%$ (84) were chronic.

At T1, joint noises were reported by $17 \%$ (57) of patients; $10 \%$ (33) were intermittent and 7\% (24) were constant. In GF at $\mathrm{T} 2$, joint noises were present in $12 \%$ (10) of patients; 6\% (5) were intermittent and 6\% (5) were constant.

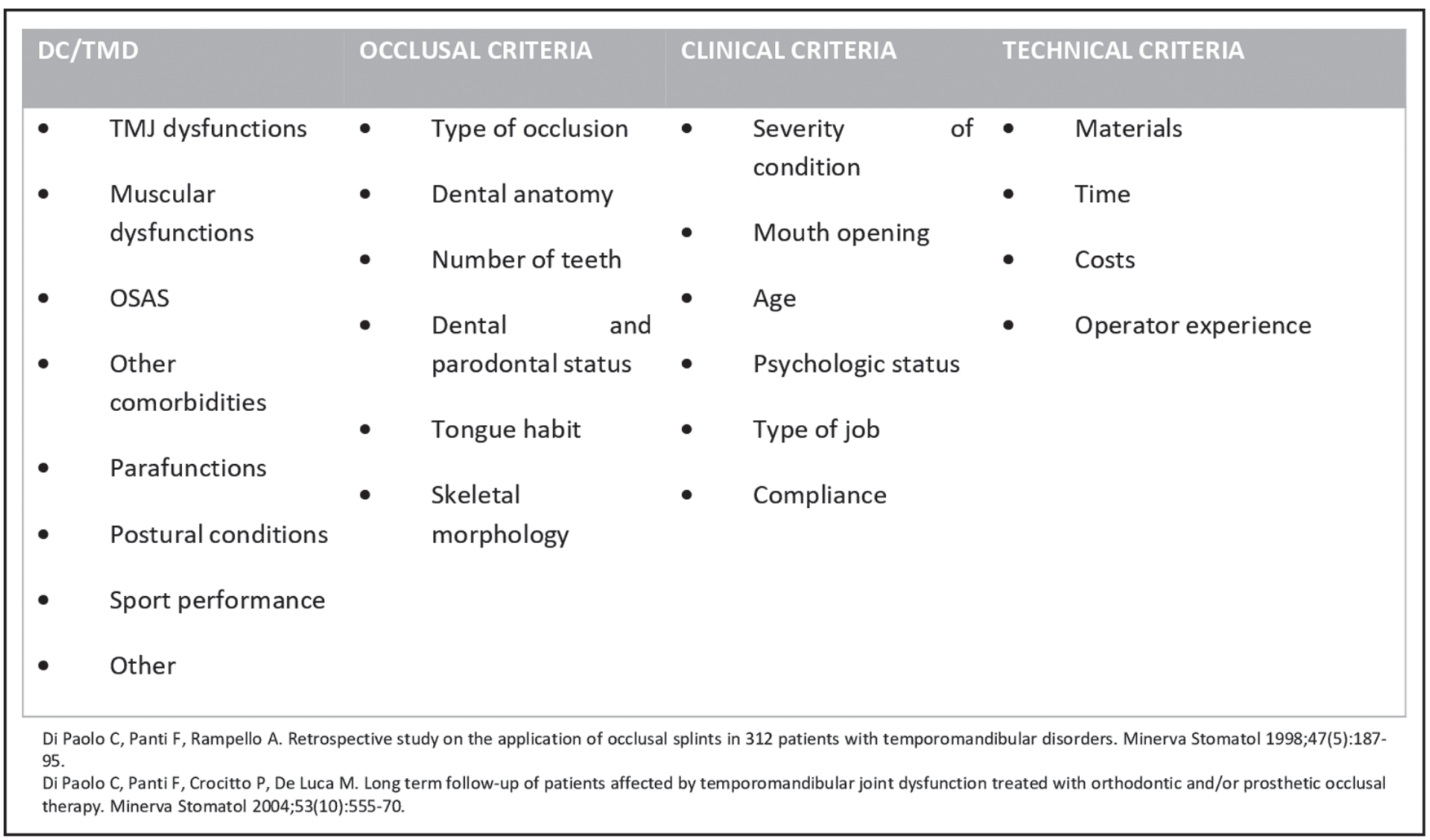

Figure 4: Additional criteria for clinical management 
Mouth opening: At T0, a mouth opening less than $40 \mathrm{~mm}$ was present in $18 \%(62)$. At $\mathrm{T} 1$ and $\mathrm{T} 2$, a mouth opening less than $40 \mathrm{~mm}$ was present in $9 \%$ of patients.

The follow-up records showed a stability of treatment outcomes in the medium term $(68 \%$ of patients) and a progressive positive change $(20 \%)$. The worsening of $13 \%$ patients in T2 was not "absolute" as compared to T1 results [Figures 6-11].

\section{EFFICIENCY: DURATION OF TREATMENTS}

The mean duration of therapy was 12 months $(\mathrm{G} 1+\mathrm{G} 2)$, with 15 months in G1 and 8 months in G2. The parameter that improved faster was the opening of the mouth. Average recovery time was $3.8 \pm 1.2$ months. Average recovery time of articular and muscular structures was $6.4 \pm 1.8$ months. For neck pain, it was $8.1 \pm 2.0$ months and for headache it was $9.6 \pm 2.4$ months.

FEASIBILITY: COMPARATION OF TREATMENT OUTCOMES BETWEEN G1 AND G2 (GENERAL SYMPTOMATOLOGY)

The statistical analysis showed no significant differences between treatment outcomes of the two groups $\left(\chi^{2}=0.1087146\right)$.

\begin{tabular}{|c|}
\hline T1 \\
\hline $\begin{array}{l}\text { HEALED/CURED (absence of all the symptoms present at the diagnosis without the } \\
\text { need of any therapeutic treatment) }\end{array}$ \\
\hline $\begin{array}{l}\text { HEALED/IMPROVED (absence of painful dysfunctional symptoms present at the } \\
\text { diagnosis and/or occasional presence of joint noises and/or occasional use of occlusal } \\
\text { splint) }\end{array}$ \\
\hline IMPROVED (at least one symptom diminished and no one worsened) \\
\hline $\begin{array}{l}\text { UNCHANGED (no modification, neither improvement or worsening, of the previous } \\
\text { state) }\end{array}$ \\
\hline WORSENED (no improvement and at least one symptom worsened) \\
\hline $\mathrm{T} 2$ \\
\hline HEALED (complete disappearance of the symptomatology present at T1) \\
\hline $\begin{array}{l}\text { HEALED/IMPROVED (disappearance of the pain symptoms present at T1 and } \\
\text { occasional presence of joint noises) }\end{array}$ \\
\hline UNCHANGED (maintenance of the result obtained at T1 ) \\
\hline WORSENED (worsening of at least 1 symptom when compared to T1 \\
\hline
\end{tabular}

Figure 5: Qualitative measures

\section{Discussion}

Occlusal splints are the most common therapy among conservative treatments, and they share common therapeutic principles, such as reduction of joint load, muscle rest, and normalization of mandibular movements. ${ }^{[5,11]}$ However, there are no clearly defined guidelines in terms of choice and applied methods of occlusal devices in scientific literature. A recent metaanalysis conducted by Kuzmanovic Pficer ${ }^{[12]}$ examined several studies performed on occlusal splints over the years. Comparing their methodologies and results with those of the current research, with a few constraints, it has been possible to make the following considerations. In the studies taken into account, only stabilization splint (SS) has been administered, which is one of the favorite therapies for TMD. SS was applied to all patients regardless of the pathology. Research studies using the same evaluation scales (such as VNS or visual analog scale) of the study were evaluated. Such studies showed an improvement of the algic symptomatology records and turned out superimposable to ours, but far the following: samples were modest and the improvement was shown especially in the short term. Moreover, as mentioned in the meta-analysis, there were no specific indications on the kind of symptoms (such as joint pain or muscular pain) that has actually improved and the rate of improvement. Being the samples nonhomogeneous, it has not been possible to always evaluate the type of (patient and) pathology benefiting from this type of occlusal splint (SS). Positive results could be due to the fact that the type of patients most often sampled is the one having neuromuscular disorders, which usually benefits from the SSs. Furthermore, the mean duration of treatments and the mandibular functionality (reduction/absence of joint noises and quality of the mandibular movement) after therapy, except for maximum mouth opening, were not evaluated; only in a few articles was the action of splints on the main comorbidities (headache/neck pain) assessed. Joint

Table 1: Personal data, type of DC/TMD disorder, and type of splint

\begin{tabular}{ll}
\hline Personal data & Female $83 \%$ (281 patients); male $17 \%$ (56 patients). The average age was 37.16 years \\
Type of DC/TMD disorder & Disc dislocation with reduction $31 \%(104)$ \\
& Myalgia $30 \%(102)$ \\
& Disc dislocation without reduction with limited mouth opening $13 \%$ (44) \\
& Degenerative diseases $13 \%(44)$ \\
& Arthralgia $7 \%(24)$ \\
& Disc dislocation without reduction without limited opening 6\% (19) \\
& DI.TRA 74\% (251) \\
Type of splint & DI.TRA AND RA.DI.CA $12 \%(40)$ \\
& RA.DI.CA $\%$ (29) \\
& MICHIGAN SPLINT 5\% (17) \\
\end{tabular}


pain and disorders should be assessed and treated in a targeted way, according to the rationale of this study. However, there is no analysis of patients with joint dysfunction and treated with specific $\mathrm{OA}$, with a medium long-term follow-up, in scientific literature. The use of specific splint types for joint disorders (disc dislocation with or without reduction) is hardly considered in the literature, except for the study by Greene and Menchel, ${ }^{[7]}$ Madani and Mirmortazavi, ${ }^{[13]}$ and Williamson. ${ }^{[14,15]}$ The last ones reported a consistent improvement of pain $(81 \%)$ and a reduction of joint noises, using anterior repositioning splints for disc dislocation with reduction, but the sample was small and there was not a follow-up group. ${ }^{[13,14,16]}$

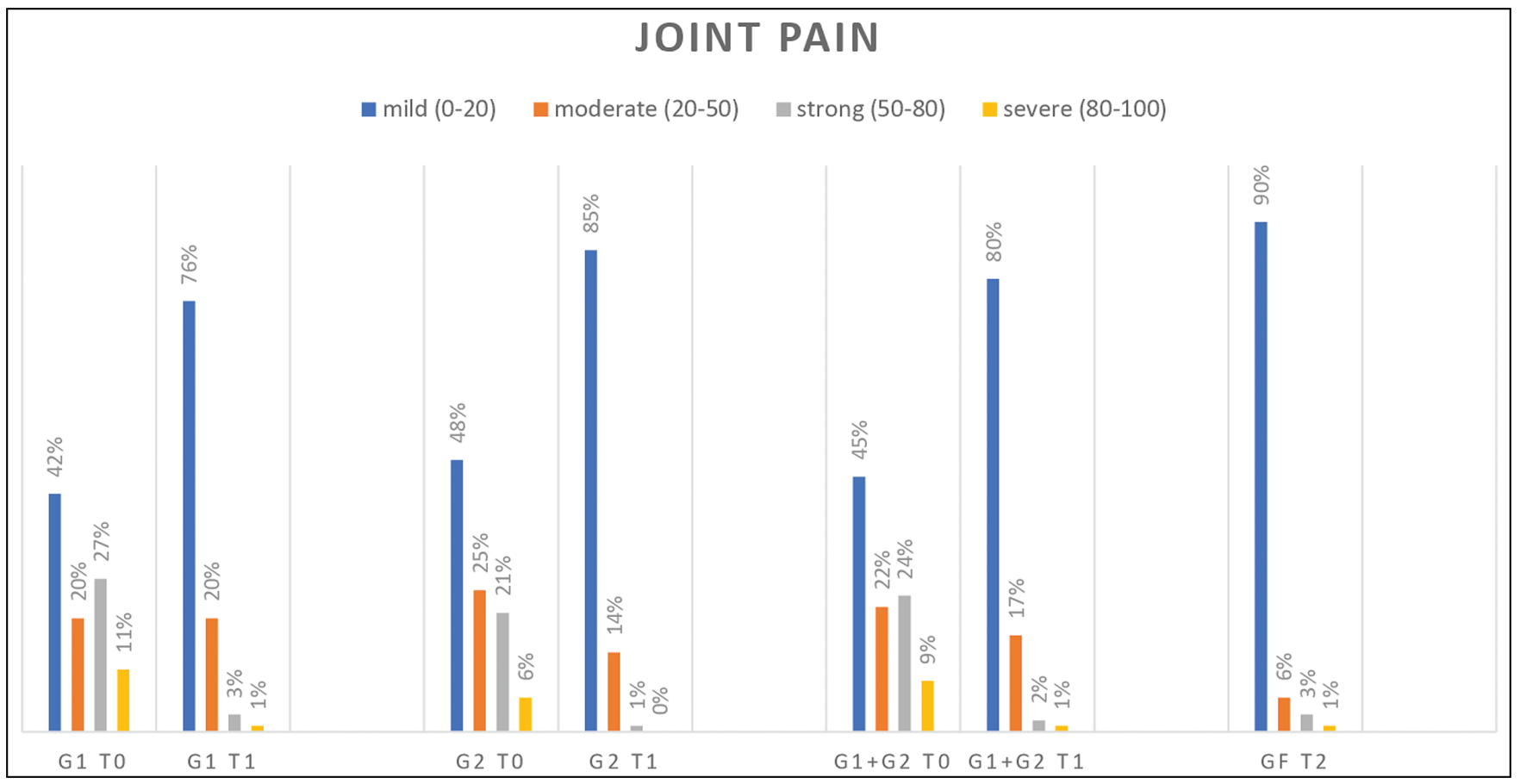

Figure 6: Patients with joint pain (\%) for each VNS category

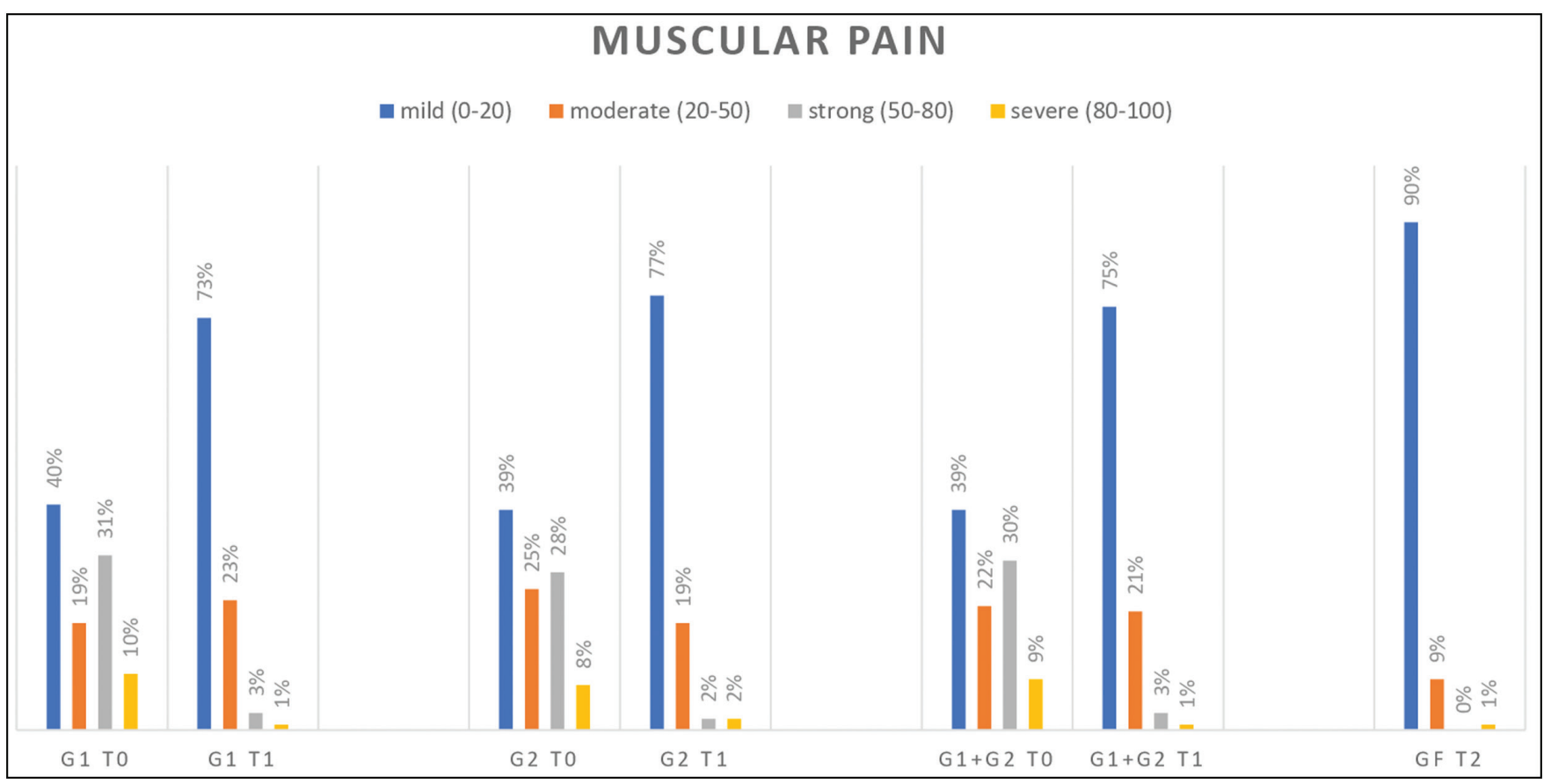

Figure 7: Patients with muscular pain (\%) for each VNS category 
Moreover, not all these studies follow a diagnostic classification, scientifically validated (such as $\mathrm{DC} /$ TMD), and there is no "specificity" of therapy as to patient and pathology. In fact, there are no "systematic" therapies and it appears to be the tendency to look an "all-in-one" device for all pathologies and patients, creating a "therapeutic funnel," except for the studies mentioned earlier and our previous studies. ${ }^{[7-10,13,14]}$

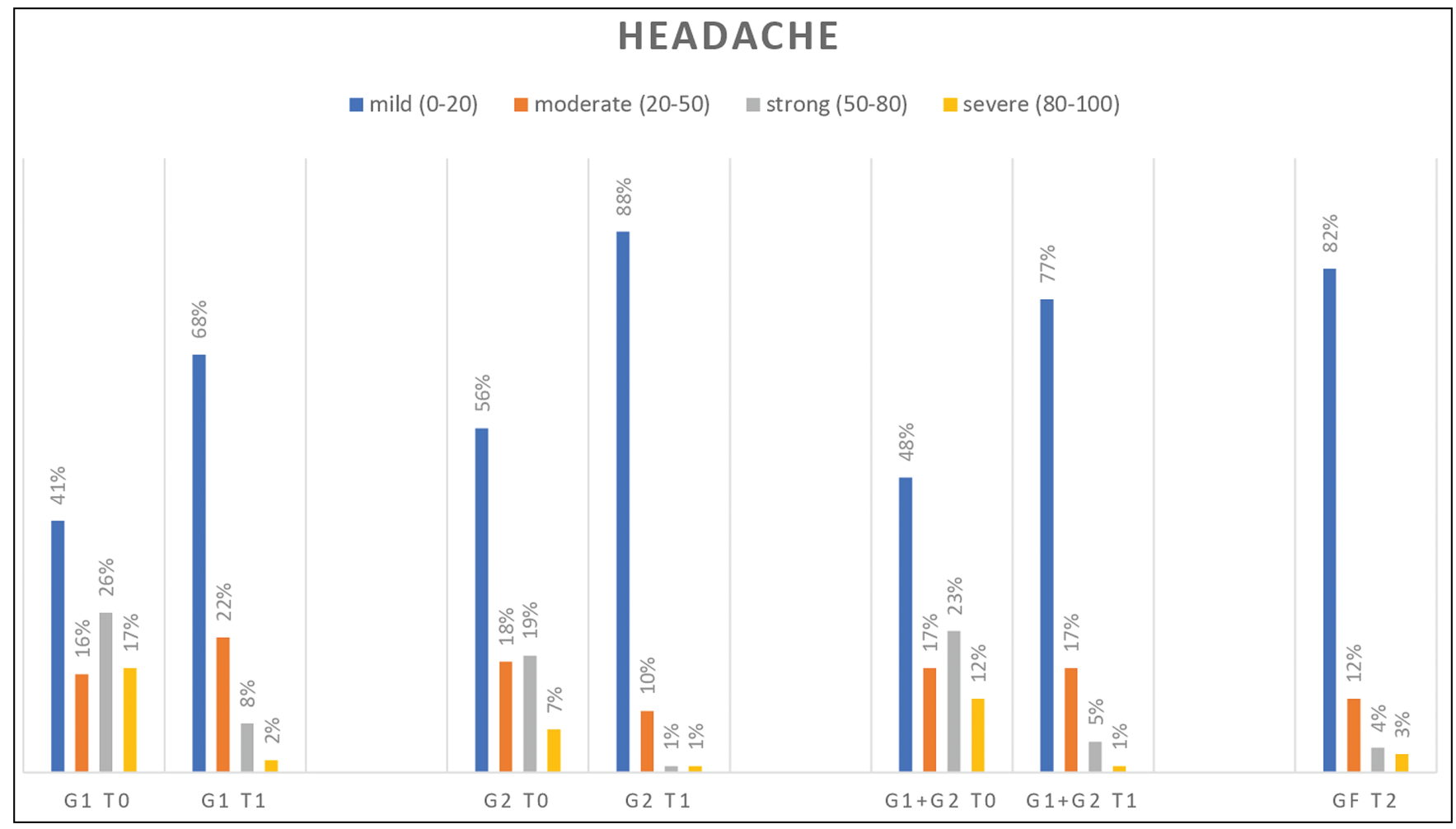

Figure 8: Patients with headache (\%) for each VNS category

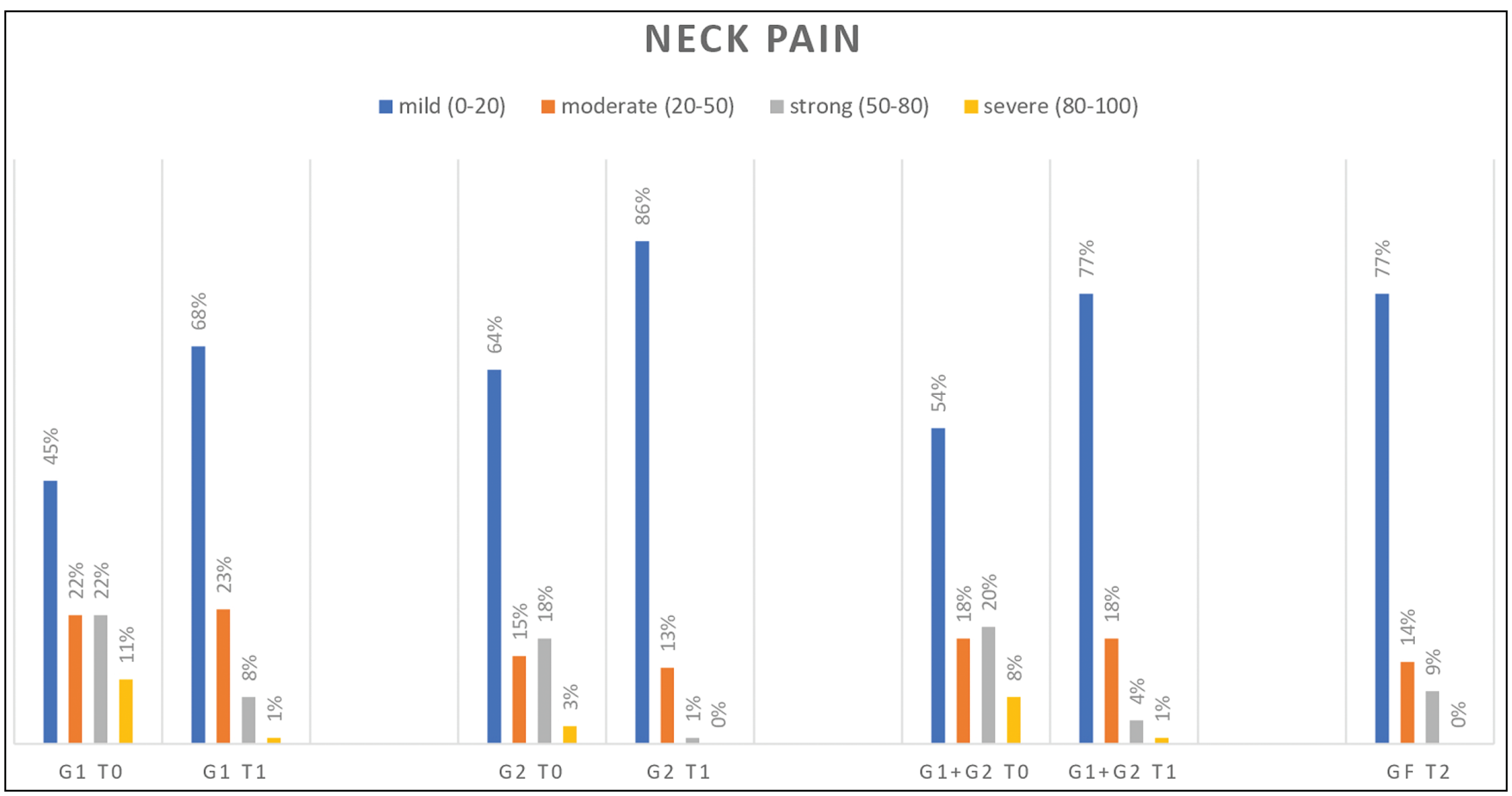

Figure 9: Patients with neck pain (\%) for each VNS category 
Furthermore, scientific literature does not give any indications on splint management, and the authors of this study believe it should be customized and consider any specific clinical condition.

In light at the foregoing, it is high time a systematic protocol was used, having regard to the positive trend of results in all parameters analyzed by this study (efficacy and efficiency) and the feasibility of the procedure, which starting from the diagnosis allows to give around to the choice and the subsequent management of the most appropriate occlusal appliance for the specific clinical condition.

Our indications on the type of splint (DI.TRA/RA.DI. CA rather than Farrar Splint or MFDA for example) are not rigid, but they derive from the integration

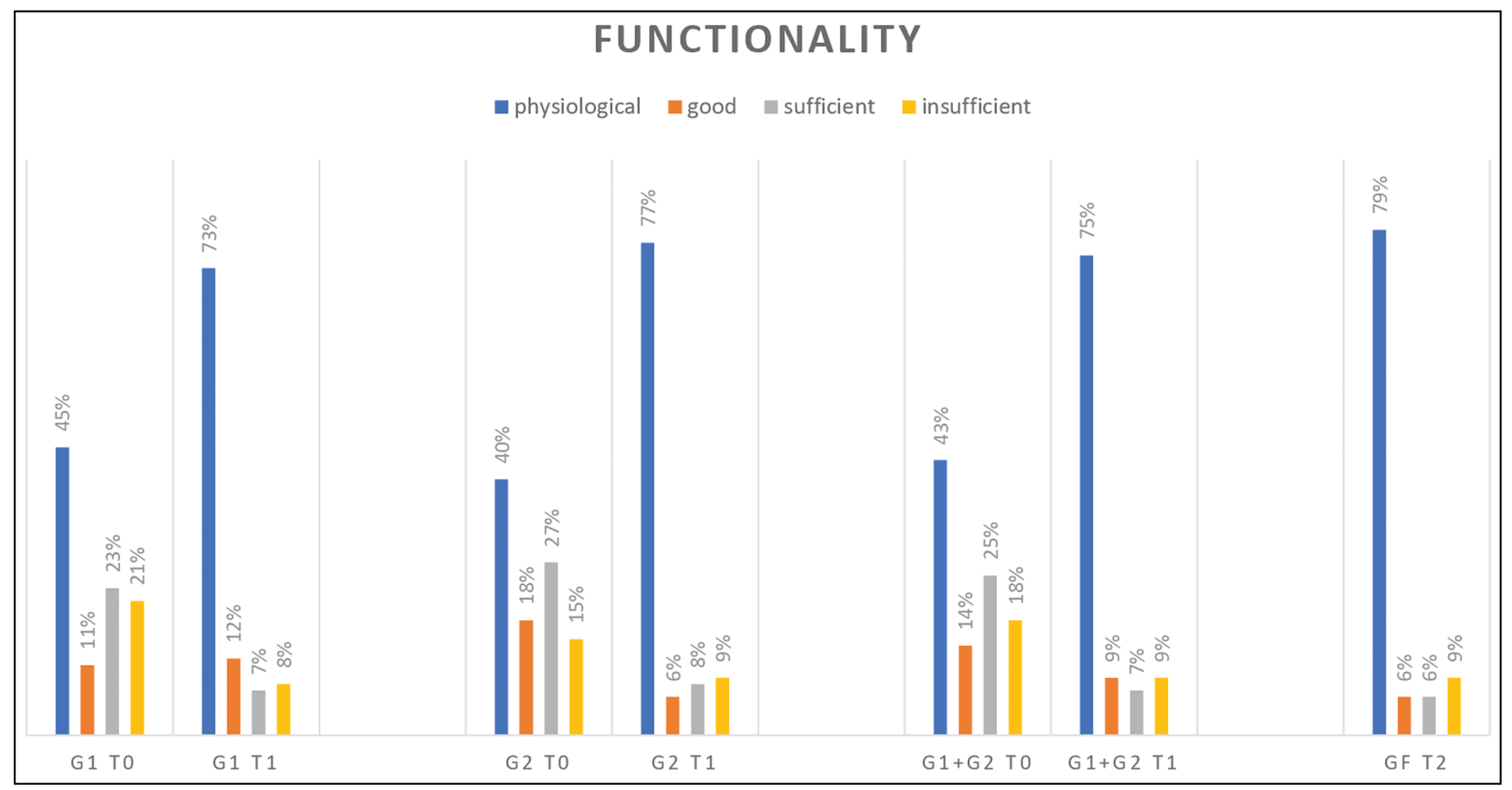

Figure 10: Patients with mandibular functionality (\%) for each VNS category

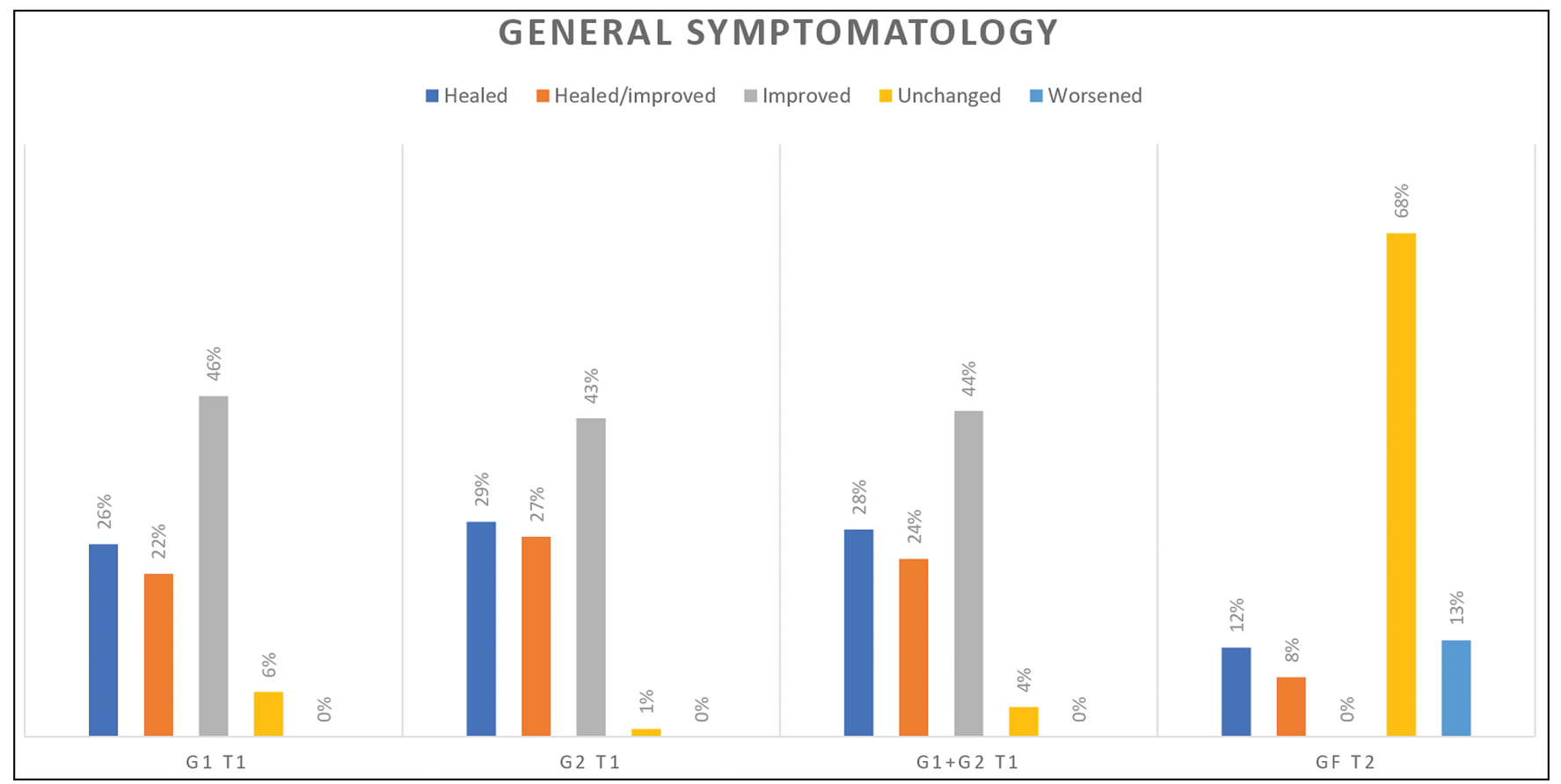

Figure 11: Patients with general symptomatology (\%) for each VNS category 
of scientific literature with our clinical experience. However, the authors maintain that for each type of disorder there is a different category of splint (flat plane splint/repositioning splint, distant splint), which should be kept into consideration for a therapy to be effective and efficient also in the medium-long term. This position differs from what emerges in the study in which the positive results are basically recorded in the short term. ${ }^{[12]}$

However, the authors suggest that an accurate choice of oral devices is not enough to guarantee any therapeutical effectiveness, in the event that there are no correct management protocol for treatment of patients and their specific condition. A correct management is also highly advisable to avoid any complications secondary to splint therapy such as those resulting from any prolonged use. Also in line with other studies, our study revealed lower trend of occlusal modification due to the progressive reduction of the use of the splint. ${ }^{[13,17,18]}$

In addition, the authors recommend tailor-made laboratory techniques for the construction of the splint and tailor-made instructions in management with specific and sequential phases for the whole care span. ${ }^{[19-21]}$

The validity of protocol was also assessed as to its feasibility. Therefore, by comparing homogeneous samples stemming from two different structures, the authors made an appraisal of the aforesaid model of treatment and their applicability by any operator. The results were overlapping and not statistically relevant. This implies that the proposed protocol leads to results depending on the operator to a limited extent, in terms of effectiveness of therapy. The only discrepancy, actually significant, between the two groups was the different therapy lapse (efficiency). This is due to a likely greater efficiency of the single operator and/or to a greater difficulty in managing appointments in the public structure.

Finally, it is evaluated whether occlusal appliances may be an effective treatment modality for some TMDs due to their potential to serve as an elaborate placebo, more than any specific therapeutic mechanism.

\section{LIMITATION OF THE STUDY}

A limitation of this study was that the protocol only contemplates selected occlusal devices and does not concern all type of splints.

\section{FUTURE SCOPE}

The authors aimed to conduct also a long-term follow-up study in order to assess the stability of TMD treatments over time and to compare splint therapy outcomes with those of other conservative treatments. ${ }^{[22]}$

\section{Conclusions}

In light of the aforementioned observations, we believe that this setting of protocol, together with an adequate choice and management of the occlusal appliances, proved to be well effective and quite efficient, less operator dependent, and integrated with indications of literature.

It is highly advisable that, as for the diagnosis, specific therapeutical guidelines are developed based on current knowledge and on critical evaluation of short-, medium-, and long-term clinical results of conservative protocols, so that the care of dysfunctional patients (adults and growing) could be predictable.

FINANCIAL SUPPORT AND SPONSORSHIP

Nil.

\section{CONFLICTS OF INTEREST}

There are no conflicts of interest.

\section{REFERENCES}

1. Luther F. TMD and occlusion part I. Damned if we do? Occlusion: the interface of dentistry and orthodontics. Br Dent J 2007;202:E2.

2. Tomoyasu Y, Higuchi H, Mori M, Takaya K, Honda Y, Yamane A, et al. Chronic orofacial pain in dental patients: Retrospective investigation over 12 years. Acta Med Okayama 2014;68:269-75.

3. Schiffman E, Ohrbach R, Truelove E, Look J, Anderson G, Goulet JP, et al.; International RDC/TMD Consortium Network, International association for Dental Research; Orofacial Pain Special Interest Group, International Association for the Study of Pain. Diagnostic Criteria for Temporomandibular Disorders (DC/TMD) for clinical and research applications: Recommendations of the international RDC/TMD consortium network* and orofacial pain special interest group $\dagger$. J Oral Facial Pain Headache 2014;28:6-27.

4. Wieckiewicz M, Boening K, Wiland P, Shiau YY, ParadowskaStolarz A. Reported concepts for the treatment modalities and pain management of temporomandibular disorders. J Headache Pain 2015;16:106.

5. Ebrahim S, Montoya L, Busse JW, Carrasco-Labra A, Guyatt GH; Medically Unexplained Syndromes Research Group. The effectiveness of splint therapy in patients with temporomandibular disorders: A systematic review and metaanalysis. J Am Dent Assoc 2012;143:847-57.

6. Hasegawa Y, Kakimoto N, Tomita S, Fujiwara M, Ishikura R, Kishimoto $\mathrm{H}$, et al. Clinical study of splint therapeutic efficacy for the relief of temporomandibular joint discomfort. J Craniomaxillofac Surg 2017;45:1772-7.

7. Greene CS, Menchel HF. The use of oral appliances in the management of temporomandibular disorders. Oral Maxillofac Surg Clin North Am 2018;30:265-77.

8. Cascone P, Di Paolo C. Patologia dell'articolazione temporomandibolare. Dall'eziopatogenesi alla terapia. Torino: UTET; 2004; pp. 174-5.

9. Di Paolo C, Panti F, Rampello A. [Retrospective study on the application of occlusal splints in 312 patients with temporomandibular disorders]. Minerva Stomatol 1998;47:187-95. 
10. Di Paolo C, Panti F, Crocitto P, De Luca M. Long term follow-up of patients affected by temporomandibular joint dysfunction treated with orthodontic and/or prosthetic occlusal therapy. Minerva Stomatol 2004;53:555-70.

11. Akbulut N, Altan A, Akbulut S, Atakan C. Evaluation of the $3 \mathrm{~mm}$ thickness splint therapy on temporomandibular joint disorders (TMDs). Pain Res Manag 2018;2018: 3756587.

12. Kuzmanovic Pficer J, Dodic S, Lazic V, Trajkovic G, Milic N, Milicic B. Occlusal stabilization splint for patients with temporomandibular disorders: Meta-analysis of short and long term effects. PLoS One 2017;12:e0171296.

13. Madani AS, Mirmortazavi A. Comparison of three treatment options for painful temporomandibular joint clicking. J Oral Sci 2011;53:349-54.

14. Williamson EH. Temporomandibular dysfunction and repositioning splint therapy. Prog Orthod 2005;6:206-13.

15. Lin SL, Wu SL, Ko SY, Yen CY, Yang JW. Effect of flat-plane splint vertical thickness on disc displacement without reduction: A retrospective matched-cohort study. J Oral Maxillofac Surg 2017;75:1627-36.

16. Liu MQ, Lei J, Han JH, Yap AU, Fu KY. Metrical analysis of disc-condyle relation with different splint treatment positions in patients with TMJ disc displacement. J Appl Oral Sci 2017;25:483-9.

17. Conti PC, Miranda JE, Conti AC, Pegoraro LF, Araújo Cdos R. Partial time use of anterior repositioning splints in the management of TMJ pain and dysfunction: A one-year controlled study. J Appl Oral Sci 2005;13:345-50.

18. Ramfjord SP, Ash MM. Reflections on the Michigan occlusal splint. J Oral Rehabil 1994;21:491-500.

19. Michelotti A, Iodice G, Vollaro S, Steenks MH, Farella M. Evaluation of the short-term effectiveness of education versus an occlusal splint for the treatment of myofascial pain of the jaw muscles. J Am Dent Assoc 2012;143:47-53.

20. De la Torre Canales G, Manfredini D, Grillo CM, GuardaNardini L, Machado Gonçalves L, Rizzatti Barbosa CM. Therapeutic effectiveness of a combined counseling plus stabilization appliance treatment for myofascial pain of the jaw muscles: A pilot study. Cranio 2017;35:180-6.

21. Hasanoglu Erbasar GN, Alpaslan C, Eroglu Inan G. Can an NTI-tss device be effective as a first-line therapy in patients with TMD myofascial pain? J Oral Rehabil 2017;44:589-93.

22. Celakil T, Muric A, Gökcen Roehlig B, Evlioglu G. Management of pain in TMD patients: Bio-oxidative ozone therapy versus occlusal splints. Cranio 2019;37:85-93. 